\title{
Mean-field games and two-point boundary value problems
}

\author{
Thulasi Mylvaganam and Dario Bauso and Alessandro Astolfi
}

\begin{abstract}
A large population of agents seeking to regulate their state to values characterized by a low density is considered. The problem is posed as a mean-field game, for which solutions depend on two partial differential equations, namely the Hamilton-Jacobi-Bellman equation and the Fokker-PlankKolmogorov equation. The case in which the distribution of agents is a sum of polynomials and the value function is quadratic is considered. It is shown that a set of ordinary differential equations, with two-point boundary value conditions, can be solved in place of the more complicated partial differential equations associated with the problem. The theory is illustrated by a numerical example.
\end{abstract}

\section{INTRODUCTION}

A population of dynamic agents, assumed to be indistinguishable, is considered. We assume that each agent's dynamics is given by a linear stochastic differential equation (SDE) driven by a Brownian motion and under the influence of a control and an adversarial disturbance. Furthermore, the population can be described by a density distribution and each of the agents seeks to select its control to minimise a certain cost functional, which may in general be functions of the state and of the density distribution, which is referred to as a mean-field term. This problem, which includes both the individual agent and the behaviour of the population is known as a mean-field game.

Related literature on mean-field games. The theory of mean-field games was introduced by Lasry and Lions in [18] and by M.Y. Huang, P. E. Caines and R. Malhamé in [15], [13], [14]. The framework provided by mean-field differential games is particularly useful in the study of differential games with a large number of players. In essence, by using tools from differential game theory, mathematical physics, and $H_{\infty}$-optimal control, mean-field dynamical games provide a modeling framework that allows to study the interaction between a mass of players and each individual. Such problems arise in several application domains such as economics, physics, biology, and network engineering, to mention a few [3], [4], [7], [12], [14], [17], [20], [23].

This work was supported by the 2012 "Research Fellow" Program of the Dipartimento di Matematica, Università di Trento and by PRIN 20103S5RN3 "Robust decision making in markets and organizations, 20132016". D. Bauso is currently academic visitor at the Department of Electrical and Electronic Engineering, Imperial College, UK.

T. Mylvaganam is with Department of Electrical and Electronic Engineering, Imperial College London, London SW7 2AZ, UK, thulasi.mylvaganam06@imperial.ac.uk

D. Bauso is with DICGIM, Università di Palermo, I-90128 Palermo, Italy dario.bauso@unipa.it

A. Astolfi is with Department of Electrical and Electronic Engineering, Imperial College London, London SW7 2AZ, UK and the Dipartimento di Ingegneria Civile e Ingegneria Informatica, Università di Roma "Tor Vergata", Via del Politecnico, 100133 Roma, Italy, a.astolfi@ic.ac.uk
Obtaining the solution of a mean-field game boils down to solving a system of two coupled partial differential equations (PDEs), namely the Hamilton-Jacobi-Bellman (HJB) equation and the Fokker-Planck-Kolmogorov (FPK) equation, which describes the density of the players [18], [22]. Closed-form solutions to these PDEs in terms of mean-field equilibria cannot generally be found, unless the problem has a linear-quadratic structure, see [5].

We consider a population of "crowd-averse" dynamic agents, i.e. the agents are such that they seek to regulate their state to values characterized by a low density, thus avoiding "crowded" states. Similar problems arise naturally in social sciences: the states represent opinions, the dynamics represent the propagation of these opinions, and crowdaverse attitudes capture the agents' willingness to escape consensus and seek dissensus (in the space of opinions). The crowd-averse behaviour is described by a cost functional which involves quadratic penalty on control and mean-field term involving the density of the players. We analyse the case where the initial distribution is a sum of polynomial terms and the value function is quadratic. Similar problems have been considered in [8], [9]. In this paper, a general problem in which the distribution is a sum of polynomial terms, which can be interpreted as a Taylor approximation of the distribution, is considered.

Main result. The main result of the paper is that the PDEs associated with the mean-field game are transformed into two sets of ordinary differential equations (ODEs) with twopoint boundary value conditions. The first set of equations is obtained from the HJB equation, and the second set is derived from the FPK equation. Thus a pair of coupled PDEs is transformed into two sets of ODEs, for which solutions can be readily obtained.

The remainder of the paper is structured as follows. In Section II we introduce the problem for which the main motivation is discussed in section III. The main results are presented in Section IV followed by stochastic stability analysis in Section V. In Section VI a numerical example illustrating the theory is given. Finally in Section VII we provide some conclusions and directions for future work.

Notation. We denote with $(\Omega, \mathcal{F}, \mathbb{P})$ a complete probability space. Let $\mathcal{B}$ be a finite-dimensional Brownian motion defined on this probability space. Let $\mathbb{F}=\left(\mathcal{F}_{t}\right)_{t \geq 0}$ be its natural filtration augmented by all the $\mathbb{P}$-null sets (sets of measure-zero with respect to $\mathbb{P}$ ). We use $\partial_{x}$ and $\partial_{x x}^{2}$ to denote the first and second partial derivatives with respect to $x$, respectively. 


\section{PROBlem SET-UP}

Consider a game with an infinite number of homogeneous players. The players are often referred to as agents and since they are homogeneous, they are indistinguishable. For each player let $x_{0}$ be its initial state, which is realised according to the probability distribution $m_{0}$. The state of the player at time $t$, denoted by $x_{t} \in \mathbb{R}$, evolves according to a controlled stochastic process over a finite horizon $T>0$, i.e. it satisfies the dynamics

$$
d x_{t}=\left[\alpha x_{t}+\beta u_{t}\right] d t+\sigma\left[x_{t} d \mathcal{B}_{t}+\zeta_{t} d t\right],
$$

where $u_{t} \in \mathbb{R}$ is the control input, $\mathcal{B}_{t} \in \mathbb{R}$ is a Brownian motion, which is independent of the initial state $x_{0}$ and independent across players and time. The constants $\alpha \in \mathbb{R}$, $\beta \in \mathbb{R}$ and $\sigma \in \mathbb{R}$ are parameters, and $\zeta_{t} \in \mathbb{R}$ is an adversarial disturbance.

A macroscopic description of the game is obtained by considering probability density functions on the state space:

$$
\left\{\begin{array}{l}
m: \mathbb{R} \times\left[0,+\infty\left[\rightarrow \left[0,+\infty\left[,(x, t) \mapsto m_{t}(x)\right.\right.\right.\right. \\
\int_{\mathbb{R}} m_{t}(x) d x=1 \text { for every } t,
\end{array}\right.
$$

Define now the average state distribution at time $t$ as

$$
\bar{m}_{t}:=\int_{\mathbb{R}} x m_{t}(x) d x .
$$

In the following we assume that the density distribution is polynomial in the state.

Assumption 1: The density is given by

$$
\left\{\begin{array}{l}
m_{t}(x)=a_{0 t}+\sum_{j=1}^{n} \frac{1}{j} a_{j t} x^{j}, \text { in } \mathbb{R} \times[0, T] \\
m_{0}(x)=d(x)=a_{00}+\sum_{j=1}^{n} \frac{1}{j} a_{j 0} x^{j}, \\
a_{j 0} \text { given for all } j=0, \ldots, n .
\end{array}\right.
$$

Note that the sum of polynomial terms in Assumption 1 can be interpreted as the Taylor approximation of a general distribution $m_{t}(x)$.

Each agent is given a cost functional with penalty on the final state $g(\cdot)$, stage cost function $c(\cdot)$, and quadratic penalty on the unknown disturbance, i.e.

$$
\begin{aligned}
& J\left(x_{0}, u, m, \zeta\right)=\mathbb{E}\left(g\left(x_{T}\right)\right. \\
& \left.+\int_{0}^{T} c\left(x_{t}, u_{t}, \bar{z}_{t}\right) d t-\gamma^{2} \int_{0}^{T}\left|\zeta_{t}\right|^{2} d t\right) .
\end{aligned}
$$

We consider the stage cost

$$
c\left(x_{t}, u_{t}, m\right)=a_{0 t}+a_{1 t} x+\frac{1}{2} a_{2 t} x^{2}+\frac{b}{2} u_{t}^{2} .
$$

The first three terms represents the mean-field cost and is the quadratic approximation of the density distribution, whereas $\frac{b}{2} u_{t}^{2}$, with $b>0$, accounts for a penalty on the control energy. The penalty on the final state is $g\left(x_{T}, m_{T}\left(x_{T}\right)\right)=$ $a_{0 T}+a_{1 T} x+\frac{1}{2} a_{2 T} x^{2}$, namely it is a penalty on the quadratic approximation of the state density distribution at the end of the horizon.

Remark 1: The cost function (3) is such that each of the agents are crowd-averse in the sense that they seek to regulate their states to the regions of low density. Note, however, that the agents only consider the density in the state they are in: this is known as local interaction. It may happen that all agents attempt to regulate their state to the same low-density state.

The robust mean-field game problem is defined as follows.

Problem 1: (Robust mean-field game problem) Let $\mathcal{B}$ be a one-dimensional Brownian motion defined on $(\Omega, \mathcal{F}, \mathbb{P})$. Let $x_{0}$ be independent of $\mathcal{B}$ and with density $m_{0}(x)$. Let $m_{t}^{*}$ be the optimal mean-field trajectory. The robust mean-field problem in $\mathbb{R}$ and $(0, T]$ is given by

$$
\left\{\begin{array}{c}
\inf _{\left\{u_{t}\right\}_{t}} \sup _{\left\{\zeta_{t}\right\}_{t}} J\left(x, u, m^{*}, \zeta\right) \\
d x_{t}=\left[\alpha x_{t}+\beta u_{t}+\sigma \zeta_{t}\right] d t+\sigma x_{t} d \mathcal{B}_{t}
\end{array}\right.
$$

\section{Motivations}

Problems similar to the one defined in Section II arise in different fields. However, the main motivation for the problem studied is opinion dynamics in social networks. Crowd-averse attitudes in this setting imply that the players tend to have very different opinions. This is in contrast to the "opposite" phenomena of "emulation", "mimicry" or "herd behavior".

Example 1: (Social networks) Opinion dynamics have received a significant amount of attention by many scientists. This is the study of the propagation of opinions. The main goal is to describe the time evolution of the beliefs of a large population of agents as a consequence of repeated interactions among the agents: in many cases these interactions may be over a social network, see for example [10, Sect. III] and [2]. In continuous opinion dynamics models, beliefs or opinions are represented by states, which may be scalars or vectors, that evolve according to some averaging process. These processes model the attractive nature of social influence; each opinion moves towards a convex combinations of (a subset of) other agents' current beliefs. Assuming that the underlying social network is connected, many models are such that asymptotic convergence to a consensus of the opinions can be proven. However, there are some exceptions. For example, in the models by [16] the authors introduce homophily in the form of "bounded confidence", namely the case in which agents are not influenced by beliefs that are "far" from their own. This is somewhat similar to what has been considered in [1], where competing stubborn agents are considered. Here, stubborn agents are agents that do not change their opinions but try to influence the opinions of others and these could, for example, represent leaders, political parties or media sources. For example, in [11], scaling limits results are provided: these show that, if the population of agents is homogeneous, the empirical belief distribution converges, as the population size grows large, towards the solution of a certain deterministic mean-field differential equation in the space of probability measures. These results are in the spirit of the propagation of chaos in interacting particle systems [21]. 


\section{MAIN RESUltS}

Let $v_{t}(x)$ be the (upper) value of the robust optimization problem under worst-case disturbance starting at time $t$ from state $x$. In what follows we consider quadratic value functions of the form

$$
\left\{\begin{array}{l}
v_{t}(x)=q_{0 t}+\sum_{j=1}^{2} \frac{1}{j} q_{j t} x^{j}, \text { in } \mathbb{R} \times[0, T] \\
v_{T}(x)=g\left(x_{T}, m_{T}\left(x_{T}\right)\right) \\
\quad=q_{0 T}+\sum_{j=1}^{2} \frac{1}{j} q_{j T} x^{j}=a_{0 T}+\sum_{j=1}^{2} \frac{1}{j} a_{j T} x^{j} .
\end{array}\right.
$$

Bearing this in mind, the case of a crowd-averse system where the players seek to drive their state towards state values characterized by a lower density, namely the problem described in Section II is considered.

Theorem 1: The mean-field system associated to the robust mean-field game for the crowd-averse system is described by the equations:

$$
\left\{\begin{array}{l}
\partial_{t} v_{t}+\left[-\frac{\beta^{2}}{2 b}+\left(\frac{\sigma}{2 \gamma}\right)^{2}\right]\left(\partial_{x} v_{t}\right)^{2} \\
+\alpha x \partial_{x} v_{t}+a_{0 t}+a_{1 t} x+\frac{1}{2} a_{2 t} x^{2} \\
+\frac{1}{2} \sigma^{2} x^{2} \partial_{x x}^{2} v_{t}=0, \text { in } \mathbb{R} \times[0, T[, \\
v_{T}(x)=q_{0 T}+\sum_{j=1}^{2} \frac{1}{j} q_{j T} x^{j}, \text { in } \mathbb{R}, \\
\partial_{t} m_{t}+\sum_{j=1}^{n} a_{j t}\left[\left(1+\frac{1}{j}\right)\right. \\
\quad\left(\alpha-\frac{\beta^{2}}{b} q_{2 t}+\frac{\sigma^{2}}{2 \gamma^{2}} q_{2 t}\right) x_{t}^{j} \\
\left.+\left(-\frac{\beta^{2}}{b} q_{1 t}+\frac{\sigma^{2}}{2 \gamma^{2}} q_{1 t}\right) x_{t}^{j-1}\right] \\
+a_{0 t}\left(\alpha-\frac{\beta^{2}}{b} q_{2 t}+\frac{\sigma^{2}}{2 \gamma^{2}} q_{2 t}\right) \\
-\frac{1}{2} \sigma^{2} \partial_{x x}^{2}\left(x^{2} m_{t}\right)=0, \text { in } \mathbb{R} \times[0, T[, \\
m_{0}(x)=a_{00}+\sum_{j=1}^{n} \frac{1}{j} a_{j 0} x^{j} \text { in } \mathbb{R} .
\end{array}\right.
$$

The optimal control and worst disturbance are then given by

$$
\left\{\begin{array}{l}
u_{t}^{*}=\frac{-\beta}{b} \partial_{x} v_{t} \\
\zeta_{t}^{*}=\frac{\sigma}{2 \gamma^{2}} \partial_{x} v_{t} .
\end{array}\right.
$$

The above result implies that to find the optimal control input the two coupled PDEs in (5) in $v$ and $m$ with the given boundary conditions on $v_{T}$ and $m_{0}$ must be solved. This is commonly done by iteratively solving the HJB equation for fixed $m$ and by entering the optimal $u$ obtained from (6) in the FPK equation in (5), until a fixed point in $v$ and $m$ is reached. Any solution of the above system of equations is referred to as worst-disturbance feedback meanfield equilibrium. The existence of solutions for problem (5) can be guaranteed by the following assumptions. Let $m_{0}$ be absolutely continuous with a continuous density function with finite second moment. As the integrand of the cost is convex in $u$, and concave in the disturbance $\zeta$, one gets a convex-concave stage cost function. The drift is linear and hence Lipschitz continuous because $\alpha, \beta, \sigma$ are bounded. We assume that the Fenchel transform of $c$ is Lipschitz in $(x, z)$. Finally, we assume that the function $p \longmapsto \frac{\sigma^{2}}{4 \gamma^{2}}\|p\|^{2}+H$ is strictly convex, differentiable and $\frac{\sigma^{2}}{4 \gamma^{2}}\|p\|^{2}+H$ is Lipschitz continuous. Note that this last condition is weaker than the convexity assumption on $H$. Under the above main assumptions, the existence of solution is established in Theorem 2.6 in [18]. In addition to this, as the cost is Lipschitz continuous on $m$ the solution to the asymptotic case with infinite number of players is related to the case with a finite number of players $N$ by the classical bound $\frac{1}{\sqrt{N}}$ provided in [14], [18]. The following theorem establishes that the mean-field system (5) can be replaced by a two-point boundary value problem.

Theorem 2: The mean-field system associated to the robust mean-field game for the crowd-averse system is equivalently described by the ordinary differential equations:

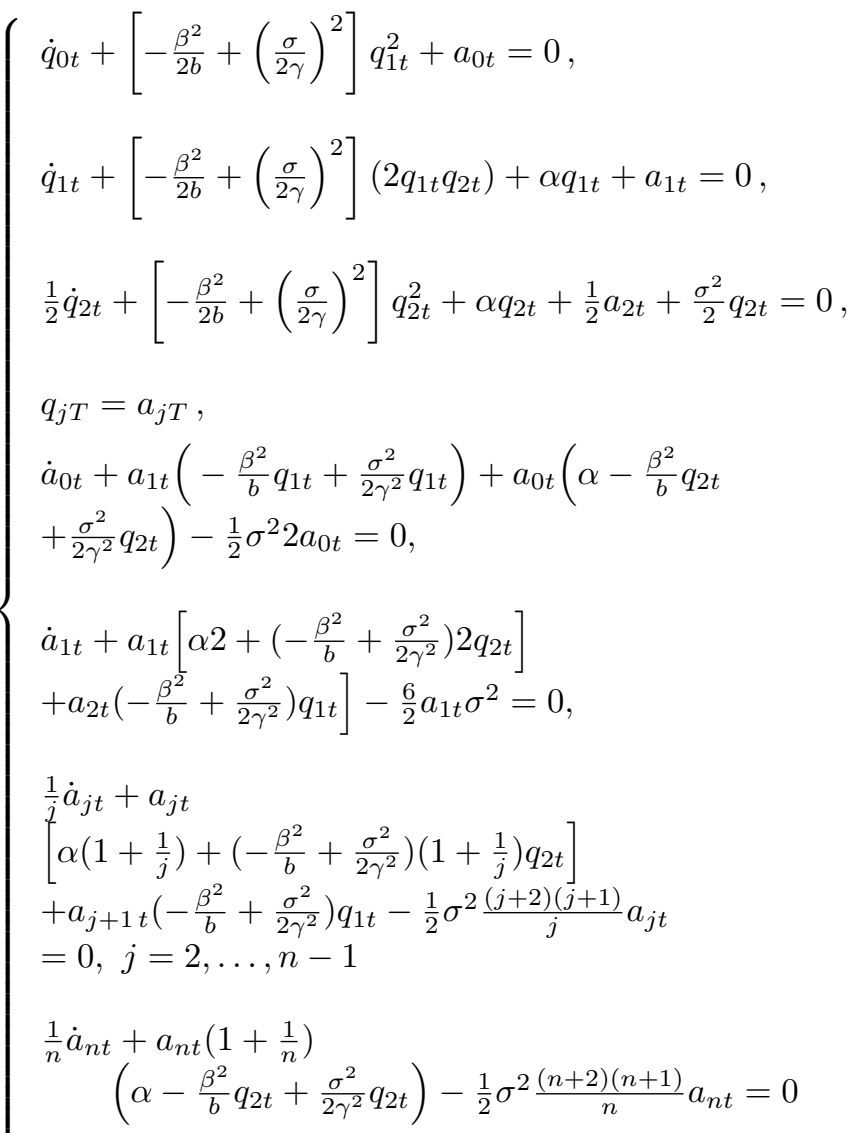

$$
\begin{aligned}
& a_{j 0} \text { given for all } j=1, \ldots, n \text {. }
\end{aligned}
$$

The optimal control and worst disturbance are then given by

$$
\left\{\begin{array}{l}
\tilde{u}_{t}=-\frac{\beta}{b}\left(q_{2 t} x_{t}+q_{1 t}\right) \\
\tilde{w}_{t}=\frac{\sigma}{2 \gamma^{2}}\left(q_{2 t} x_{t}+q_{1 t}\right)
\end{array}\right.
$$

Remark 2: The ODEs in (7) constitute a somewhat atypical two-point boundary value problem since the initial conditions $a_{j 0}$ are given for all $j$, whereas the final conditions $q_{j T}=a_{j T}$ for $j=0,1,2$ are unknown a-priori. However, by performing the change of coordinates $\tilde{q}_{j t}=q_{j t}-a_{j t}$ for $j=$ $0,1,2$, the problem can be transformed into a standard twopoint boundary value problem with final conditions $\tilde{q}_{j T}=0$. Solutions to this modified problem can be found numerically, 
for example using the shooting method, and these solutions can be used to obtain solutions to the original ODEs (7).

\section{ESTABLISHING ASYMPTOTIC STABILITY}

In this section it is shown that the stochastic differential equation describing the closed-loop system has an exponentially and asymptotically stable equilibrium. Substituting the optimal control and the worst-case disturbance (8) into the dynamics for $x_{t}$ yields the closed-loop system

$$
\begin{aligned}
& d x_{t}=\left[\alpha x_{t}+\beta u_{t}^{*}+\sigma \zeta_{t}^{*}\right] d t+\sigma x_{t} d \mathcal{B}_{t} \\
&=\left[\alpha+\left(-\frac{\beta^{2}}{b}+\frac{\sigma^{2}}{2 \gamma^{2}}\right) q_{t}\right] x_{t} d t+\sigma x_{t} d \mathcal{B}_{t}, \\
& t \in(0, T], x_{0} \in \mathbb{R}
\end{aligned}
$$

Consider now the following assumption.

Assumption 2: There exists $\kappa>0$ such that

$$
-\kappa x_{t} \geq\left[\alpha+\left(-\frac{\beta^{2}}{b}+\frac{\sigma^{2}}{2 \gamma^{2}}\right) q_{t}\right] x_{t} .
$$

With Assumption 2 the stability analysis can be performed within the framework of stochastic stability theory [19]. Consider the infinitesimal generator

$$
\mathcal{L}=\frac{1}{2} \sigma^{2} x_{t}^{2} \frac{d^{2}}{d x_{t}^{2}}-\kappa x_{t} \frac{d}{d x_{t}},
$$

and the Lyapunov function $V(x)=x^{2}$. The stochastic derivative of $V(x)$ is obtained by applying the infinitesimal generator to $V(x)$. This yields

$$
\begin{aligned}
\mathcal{L} V\left(x_{t}\right) & =\lim _{d t \rightarrow 0} \frac{\mathbb{E} V\left(x_{t+d t}\right)-V\left(x_{t}\right)}{d t} \\
& =\left[\sigma^{2}-2 \kappa\right] x_{t}^{2} .
\end{aligned}
$$

Proposition 1 ([19]): Suppose Assumption 2 holds. If $V(x) \geq 0, V(0)=0$ and $\mathcal{L} V(x) \leq-\eta V(x)$ on $Q_{\epsilon}:=$ $\{x: V(x) \leq \epsilon\}$, for some $\eta>0$, and for arbitrarily large $\epsilon$, then the origin is asymptotically stable "with probability one", and

$$
P_{x_{0}}\left\{\sup _{T \leq t<+\infty} x_{t}^{2} \geq \lambda\right\} \leq \frac{V\left(x_{0}\right) e^{-\psi T}}{\lambda}
$$

for some $\psi>0$.

From the above theorem we have the following result, which establishes exponential stochastic stability of the mean-field equilibrium.

Corollary 1: Let Assumption 2 hold. If $\left[\sigma^{2}-2 \kappa\right]<0$ then $\lim _{t \rightarrow \infty} x_{t}=0$ almost surely and

$$
P_{x_{0}}\left\{\sup _{T \leq t<+\infty} x_{t}^{2} \geq \lambda\right\} \leq \frac{V\left(x_{0}\right) e^{-\psi T}}{\lambda},
$$

for some $\psi>0$.

\section{NUMERICAL STUDIES}

A numerical example illustrating the theory is presented in this section. Consider a system consisting of $n=5422$ indistinguishable agents with dynamics (1). Furthermore, suppose each of the agents seeks to minimise a cost functional of the form (3) subject to an adversary disturbance. The initial distribution is given by $m_{0}=0.0184+0.0184 x+\frac{1}{2} 0.0373 x^{2}+$ $\frac{1}{3} 0.0019 x^{3}-\frac{1}{4} 0.0100 x^{4}$, i.e. the initial distribution is such that Assumption 1 is satisfied. The optimal control and the worst-case disturbance are then given by (8), which relies on the solution of the ODEs (7). These ODEs are solved numerically using the method discussed in Remark 2. The solution to (7) is then used to simulate the closed-loop system (1) for a discretised set of states, namely $x \in[-1,1]$. The states of the $n$ agents are initially within this set of states and their trajectories are computed over the period $[0,5]$. Simulations have been run for $\sigma=0$, i.e. without any noise or disturbances, and with $\sigma=0.05$ and $\sigma=0.1$. The remainder of the parameters are $\alpha=-0.01, \beta=0.2$, $b=0.1$ and $\gamma=1$.

Figures 1 and 2 show the solution to the two point boundary value problem (7), for the case in which $\sigma=0.05$. The solid lines in Figure 1 show the time history of the coefficients $a_{0 t}$ (top), $a_{1 t}$ (middle) and $a_{2 t}$ (bottom), whereas the dashed lines show the time histories of the coefficients $q_{0 t}$ (top), $q_{1 t}$ (middle) and $q_{2 t}$ (bottom). Note that the initial conditions on the coefficients $a_{j 0}$ and the final conditions, $q_{j T}=a_{j T}$, are satisfied for $j=0,1,2$. Figure 2 shows the time histories of the coefficents $a_{3 t}$ (top) and $a_{4 t}$ (bottom), and these are such that the initial conditions on $a_{30}$ and $a_{40}$ are satisfied. The results are similar for $\sigma=0$ and $\sigma=0.1$.

Figure 3 shows the time histories of the state of each player, when the agents use the control strategies (8), for $\sigma=0$ (top), $\sigma=0.05$ (middle) and $\sigma=0.1$ (bottom). Similarly, Figures 4, 5 and 6 show the initial (black dashed line) and final (black solid line) distribution of the agents' states for $\sigma=0, \sigma=0.05$ and $\sigma=0.1$, respectively. The dash-dotted black lines indicate the distribution of the agents at an intermediate time, namely at $t=2$. The grey lines indicate the distribution expected from the solution of the two-point boundary value problem (7) which is shown in Figures 1 and 2. Note that the distribution computed based on the evolution of the agents' states coincides well with that predicted by the solution to the two-point boundary value problems in all three cases.

The initial distribution is such that the distribution of agents is relatively low between $x=-0.5$ and $x=-1$. It is therefore expected that the agents move towards this region. This is precisely what occurs, as can be seen Figure 3.

\section{CONCLUDING REMARKS}

A problem involving a population of crowd-averse agents is formulated as a mean-field game. It is demonstrated that, under certain assumptions, a set of ODEs, forming a twopoint boundary value problem, are obtained in place of the typical PDEs associated with mean-field games. The theory 

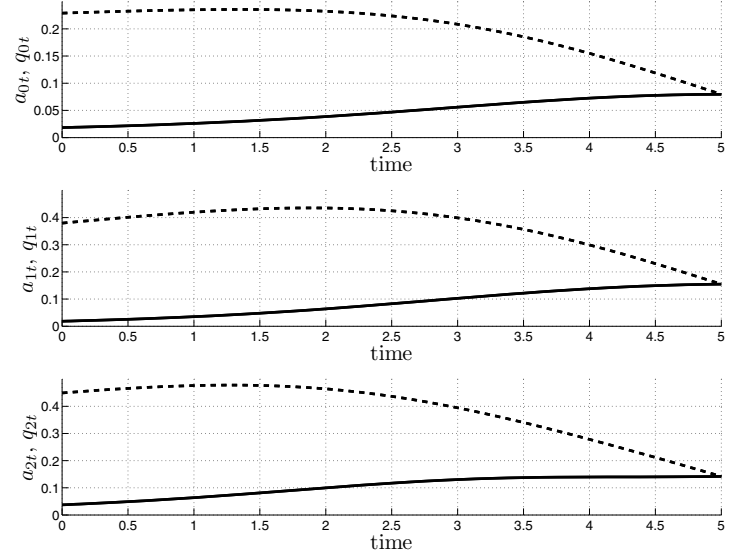

Fig. 1. Solid lines: time histories of $a_{0 t}$ (top) and $a_{1 t}$ (middle) and $a_{2 t}$ for (bottom) $\sigma=0.05$. Dashed lines: time histories of $q_{0 t}$ (top) and $q_{1 t}$ (middle) and $q_{2 t}$ (bottom) for $\sigma=0.05$
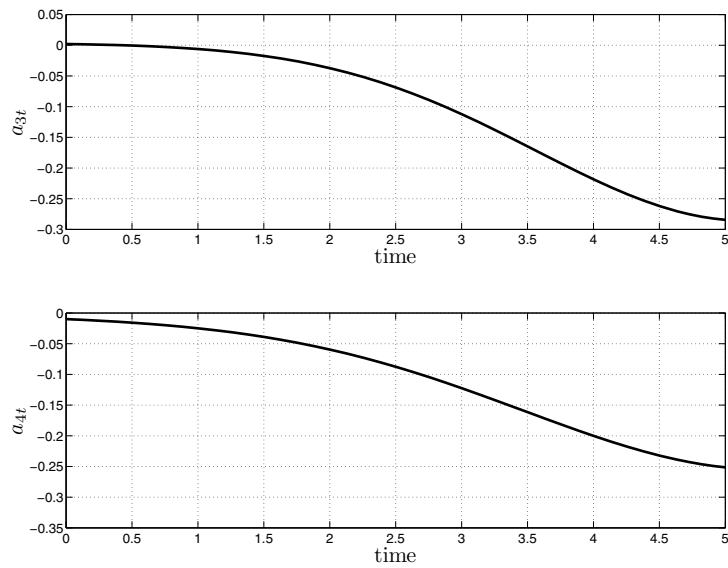

Fig. 2. Time histories of $a_{3 t}$ (top) and $a_{4 t}$ (bottom) for $\sigma=0.05$.

is illustrated by a numerical example. Directions for future work inlcludes allowing for value functions that are not quadratic. It is also if interest to consider different cost functions and consider problems in which the agents have "local" interactions only.

\section{REFERENCES}

[1] D. Acemoğlu, G. Como, F. Fagnani, and A. Ozdaglar. Opinion fluctuations and disagreement in social networks. Math. of Operation Research, vol. 38, no. 1, pp. 1-27, 2013.

[2] D. Acemoğlu, A. Ozdaglar. Opinion dynamics and learning in social networks. International Review of Economics, vol. 1, no. 1, pp. 3-49, 2011.

[3] Y. Achdou, F. Camilli, and I. Capuzzo Dolcetta. Mean field games: numerical methods for the planning problem. SIAM J. of Control Opt., vol. 50, pp. 77-109, 2012.

[4] F. Bagagiolo, and D. Bauso. Mean-field games and dynamic demand management in power grids. Dynamic Games and Applications, in print.

[5] M. Bardi. Explicit solutions of some Linear-Quadratic Mean Field Games. Network and Heterogeneous Media, 7, pp. 243-261, 2012.
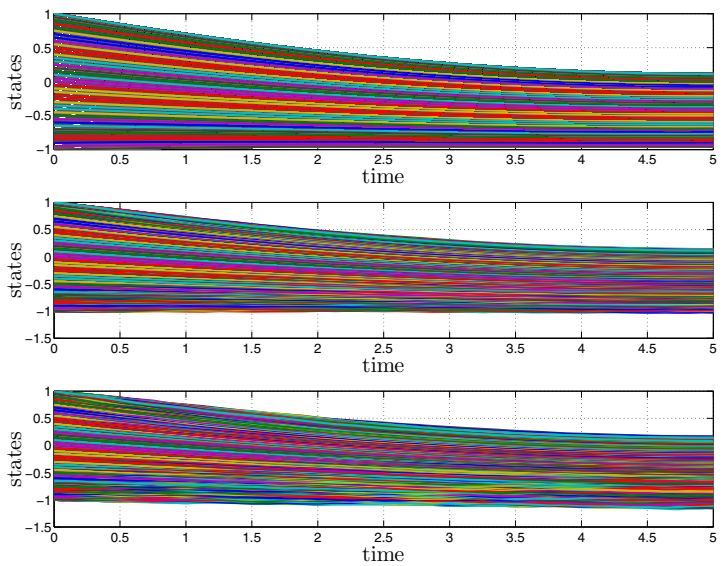

Fig. 3. Time histories of the state of each player for $\sigma=0$ (top), $\sigma=0.05$ (middle) and $\sigma=0.1$ (bottom).

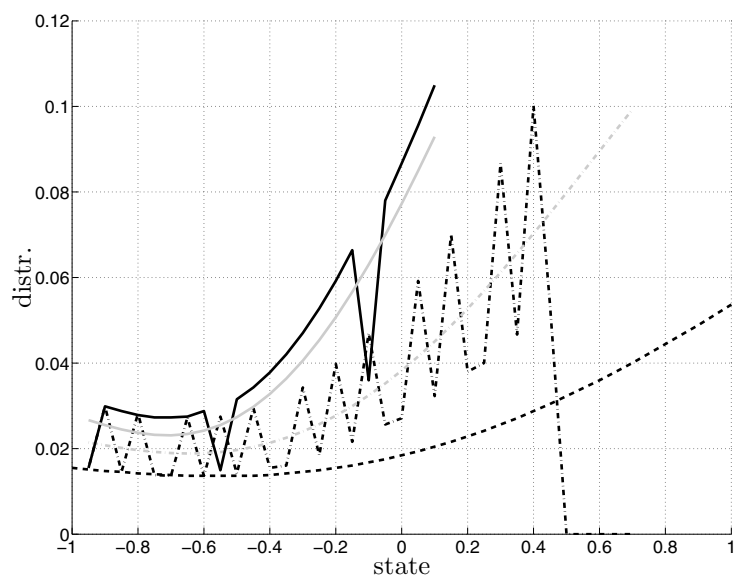

Fig. 4. Black lines: the initial (dashed line), intermediate (dash-dotted line) and final (solid line) distributions of the agents's states for $\sigma=0$ (top). Grey lines: the intermediate distribution (dash-dotted line) and final distribution (solid line) resulting from the solution of (7) for $\sigma=0$.

[6] D. Bauso, H. Tembine, and T. Başar. Robust Mean Field Games with Application to Production of an Exhaustible Resource. Proc. of 7th IFAC Symposium on Robust Control Design, Aalborg, Denmark, 2012.

[7] D. Bauso, Q. Zhu and T. Başar. Mixed Integer Optimal Compensation: Decompositions and Mean-Field Approximations. Proc. of 2012 American Control Conference, Montreal, Montreal, Canada, 2012.

[8] D. Bauso, T. Mylvaganam, and A. Astolfi. Approximate solutions for crowd-averse robust mean-field games. European Control Conference, 2013.

[9] D. Bauso, T. Mylvaganam, and A. Astolfi. A two-point boundary value formulation of a mean-field crowd-averse game. The 19th World Congress of the International Federation of Automatic Control, 2013.

[10] C. Castellano, S. Fortunato, and V. Loreto. Statistical physics of social dynamics. Rev. Mod. Phys., vol. 81, pp. 591-646, 2009.

[11] G. Como, F. Fagnani. Scaling limits for continuous opinion dynamics systems. The Annals of Applied Probability, vol. 21, no. 4, pp. 15371567.

[12] O. Gueant, J. M. Lasry, and P. L. Lions. Mean-field games and applications. Paris-Princeton Lectures, Springer, pp. 1-66, 2010.

[13] M.Y. Huang, P.E. Caines, and R.P. Malhamé. Large Population Stochastic Dynamic Games: Closed Loop Kean-Vlasov Systems and 


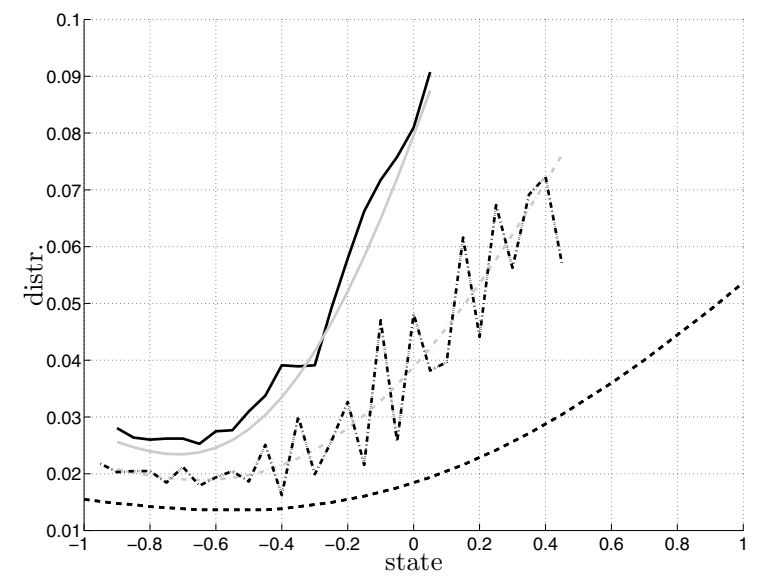

Fig. 5. Black lines: the initial (dashed line), intermediate (dash-dotted line) and final (solid line) distributions of the agents's states for $\sigma=0.05$ (top). Grey lines: the intermediate distribution (dash-dotted line) and final distribution (solid line) resulting from the solution of (7) for $\sigma=0.05$.

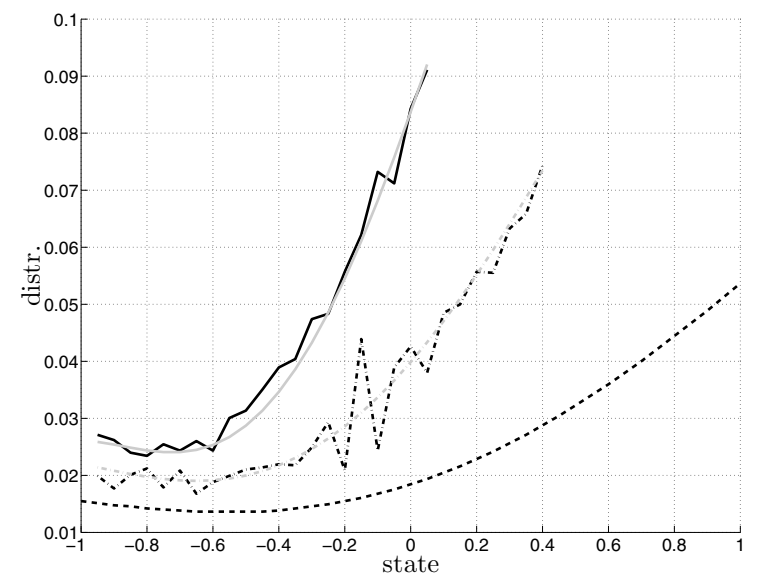

Fig. 6. Black lines: the initial (dashed line), intermediate (dash-dotted line) and final (solid line) distributions of the agents's states for $\sigma=0.1$ (top). Grey lines: the intermediate distribution (dash-dotted line) and final distribution (solid line) resulting from the solution of (7) for $\sigma=0.1$.

the Nash Certainty Equivalence Principle. Communications in Information and Systems, vol. 6, no. 3, pp. 221-252, 2006.

[14] M.Y. Huang, P.E. Caines, and R.P. Malhamé. Large population cost-coupled LQG problems with non-uniform agents: individualmass behaviour and decentralized $\epsilon$-Nash equilibria IEEE Trans. on Automatic Control, vol. 52. no. 9, pp. 1560-1571, 2007.

[15] M.Y. Huang, P.E. Caines, and R.P. Malhamé. Individual and Mass Behaviour in Large Population Stochastic Wireless Power Control Problems : Centralized and Nash Equilibrium. 42nd IEEE Conference on Decision and Control, vol. 1., pp. 98-103, 2003.

[16] U. Krause. A discrete nonlinear and non-autonomous model of consensus formation. Communications in Difference Equations, S. Elaydi, G. Ladas, J. Popenda, and J. Rakowski editors, Gordon and Breach, Amsterdam, pp. 227-236, 2000.

[17] A. Lachapelle, J. Salomon, and G. Turinici. Computation of Mean Field Equilibria in Economics. Math. Models Meth. Appl. Sci., vol. 20, pp. 1-22, 2010.

[18] J.-M. Lasry, and P.-L. Lions. Mean field games. Japanese J. Math., vol. 2, pp. 229-260, 2007.

[19] K. A. Loparo, and X. Feng. Stability of stochastic systems. The Control Handbook, CRC Press, pp. 1105-1126, 1996.
[20] R. Pesenti, and D. Bauso. Mean Field Linear Quadratic Games with Set Up Costs. Dynamic Games and Applications, vol. 3, no. 1, pp. 89-104, March 2013.

[21] A.-S. Sznitman. Topics in propagation of chaos. Springer Lecture Notes in Mathematics, vol. 1464, pp. 165-251, 1991.

[22] H. Tembine, Q. Zhu, and T. Başar. Risk-sensitive mean-field stochastic differential games. Proc. of 2011 IFAC World Congress, Milan, Italy, August 29 - September 2, 2011.

[23] H. Tembine, J. Y. Le Boudec, R. ElAzouzi, E. Altman. Mean field asymptotic of Markov decision evolutionary games. IEEE Gamenets 2009. 